In the classical theory only the difference $S_{g}-S_{s}$ is defined.

If we put $S_{g}-S_{s}=\lambda / T$ we get the same formula, the experimentally verified vapor pressure formula, for the classical as well as for the quantum theoretical expressions. But we cannot combine the classical $S_{g}$ with the quantum theoretical $S_{s}$. That would mean that we consider the atoms as distinguishable in the gas but not in the solid.
In conclusion it should be emphasized that in the foregoing remarks classical statistics is considered in principle as a part of classical mechanics which deals with individual particles (Boltzmann). The conception of atoms as particles losing their identity cannot be introduced into the classical theory without contradiction. That is possible only on the ground of the nonclassical ideas of quantum theory.

\title{
Kron's Non-Riemannian Electrodynamics
}

\author{
BANESH HOFFMANN \\ Queens College, Flushing, New York
}

\begin{abstract}
This paper discusses some aspects of Kron's tensor theories associated with the use of non-holonomic reference systems in the mathematical treatment of rotating electrical machines. The general theory is discussed in the first part, and is applied in the second part, by way of illustration, to a squirrel-cage induction motor.
\end{abstract}

\section{INTRODUCTION}

$T$ HE work of Gabriel Kron constitutes a significant enlargement of the domain of application of tensor analysis. In a previous paper ${ }^{1} \mathrm{I}$ considered that aspect of his work associated with his use of singular transformations. Here I am concerned with his discovery ${ }^{2}$ of the remarkable unifying role of non-Riemannian electrodynamics in the theory of electrical machines. Since the present paper is written for the mathematician and physicist rather than the engineer, it stresses theoretical issues to the neglect of the more practical aspects of the theory.

\section{THE GENERAL THEORY}

For a dynamical system having $n$ degrees of freedom and $n$ generalized coordinates $x^{s}$, Lagrange's equations are

$$
\frac{d}{d t}\left(\frac{\partial T}{\partial \dot{x}^{s}}\right)-\frac{\partial T}{\partial x^{s}}+\frac{\partial R}{\partial \dot{x}^{s}}=F_{s}
$$

where $x^{s}\left(\equiv d x^{s} / d t\right)$ are the generalized velocities, $T$ is the kinetic energy, $R$ is the dissipation function, ${ }^{3}$ and $F_{s}$ are the generalized impressed forces.

1 "Kron's Method of Subspaces," Quart. Applied Math. 2, 218 (1944). See also "A Mathematical Interpretation of Some Work of Gabriel Kron," published in mimeograph form by Brown University, (1943).

${ }^{2}$ See his fundamental paper "Non-Riemannian Dynamics of Rotating Electrical Machinery," Journ. Math. Phys. 13, 103 (1934). A full bibliography of Kron's publications was given by S. Austen Stigant in BEAMA Journal (August, 1948), under the title "Gabriel Kron on Tensor Analysis, A Bibliographical Record."

${ }^{3}$ This is included because of the electrical situation later. The introduction of a Rayleigh dissipation function in this way in mechanical problems is usually only an approximation.
The kinetic energy $T$ is a quadratic function of the velocities $\dot{x}^{s}$, and, under usual circumstances, a homogeneous quadratic function whose coefficients are functions of the coordinates $x^{s}$ only. We consider only this latter case and write

$$
T=\frac{1}{2} L_{s t} \dot{x}^{s} \dot{x}^{t} .
$$

For the dissipation function we may write

$$
R=\frac{1}{2} R_{s} \dot{x}^{s} \dot{x}^{t} \text {. }
$$

Here $L_{s t}, R_{s t}$ are symmetrical in $s$ and $t$ and are functions of the coordinates $x^{s}$.

It is well known that the motion of the dynamical system may be represented by that of a point, having coordinates $x^{s}$, in an $n$-dimensional Riemannian space with metric $L_{s t}$. The path of this point is given by the dynamical Eq. (1) which may be written in the tensorial form

$$
L_{s u} \dot{x}^{u},{ }_{t} \dot{x}^{t}+R_{s t} \dot{x}^{t}=F_{s},
$$

the comma denoting the covariant derivative with respect to the Christoffel symbols formed from the metric $L_{s t}$. More specifically, (4) may be written

where

$$
L_{s u} \ddot{x}^{u}+[t u, s] \dot{x}^{t} \dot{x}^{u}+R_{s t} \dot{x}^{t}=F_{s},
$$

$$
[t u, s]=\frac{1}{2}\left(\frac{\partial L_{u s}}{\partial x^{t}}+\frac{\partial L_{s t}}{\partial x^{u}}-\frac{\partial L_{t u}}{\partial x^{s}}\right) .
$$

Maxwell showed that the Lagrangian dynamical equations could be applied to electrodynamical problems if in addition to mechanical one included electrical degrees of freedom, say $m$ of them, described by $m$ coordinates $x^{a}$ measuring the total amount of charge transported across a given point in each independent 
electrical circuit from an initial time $t_{0}$ to any time $t$. The $m$ quantities $\dot{x}^{a}$, which in dynamics would be called generalized velocities, are here the currents $i^{a}$ in the various independent circuits.

For certain electrodynamical systems the kinetic energy has the form

$$
T=\frac{1}{2} L_{a b} \dot{x}^{a} \dot{x}^{b}+\frac{1}{2} L_{s t} \dot{x}^{s} \dot{x}^{t},
$$

where $a, b$ refer to electrical degrees of freedom and $s, t$ to mechanical. Maxwell showed that in macroscopic investigations mixed terms of the type $\frac{1}{2} L_{a s} \dot{x}^{a} \dot{x}^{8}$ will not appear in $T$. The quantities $L_{a b}$ measure the self and mutual inductances of the various electrical circuits. ${ }^{4}$ The $L_{s t}$ measure such quantities as masses and moments of inertia.

The electrical dissipation tensor, which we may denote by $R_{a b}$, measures the electrical resistances in the various circuits. The mechanical dissipation tensor $R_{s t}$ refers to mechanical dissipation of energy; but since a mechanical dissipation tensor can adequately represent an actual situation only under special circumstances, we shall here take the mechanical dissipation tensor to be zero.

The $L$ 's and $R$ 's whether electrical or mechanical, depend only on the mechanical coordinates $x^{s}$ and do not contain the electrical coordinates $x^{a}$. Nor do they contain the generalized velocities $\dot{x}^{a}, \dot{x}^{s}$. (When saturation and analogous phenomena are taken into account this is no longer true, but such matters are outside the scope of the present discussion.)

In geometry, the permanent absence of specific coordinates from the components of all geometric objects is called a cylinder condition. Thus in the $(m+n)-$ dimensional space having coordinates $x^{\alpha}(\alpha=a, s)$ there is a cylinder condition associated with the $m$ electrical coordinates $x^{a}$.

Using Greek indices $\alpha, \beta, \cdots$ for the range $(a, s)$, we may write the Lagrange-Maxwell electrodynamical equations in the same form as (1), (4), or (5), with these Greek indices replacing the Latin indices there used. Thus the behavior of an electrodynamical system may be represented by the motion of a point $x^{\alpha}$ in an $(m+n)$-dimensional Riemannian space in which $m$ of the coordinates obey a cylinder condition. The metric is $L_{\alpha \beta}$, where

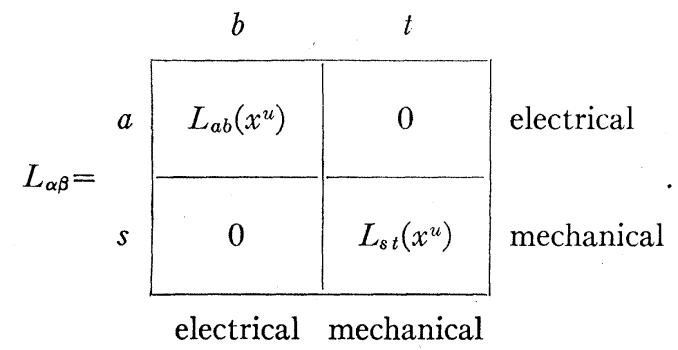

${ }^{4}$ That is, provided the $\dot{x}^{a}$ 's represent simple currents in individual circuits. Naturally, if a general transformation were made on the $\dot{x}^{a}$ 's the $L_{a b}$ 's would lose this simple physical significance. An analogous situation exists in the purely mechanical case.
The dissipation tensor $R_{\alpha \beta}$ here has the corresponding form

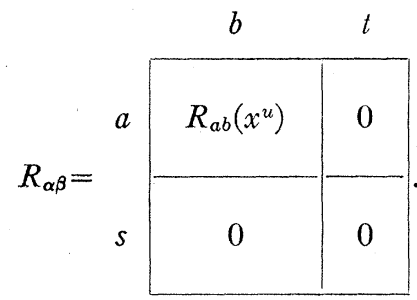

In the theory of rotating electrical machines one has occasion to introduce a non-holonomic reference system of a special type. The transformation leading to this non-holonomic reference frame is always of the restricted form

$$
\left.\begin{array}{rl}
d \bar{x}^{b} & =c_{a}^{b}\left(x^{s}\right) \cdot d x^{a} \\
\bar{x}^{s} & =\bar{x}^{s}\left(x^{t}\right)
\end{array}\right\}
$$

in which the mechanical coordinates transform holonomically while the electrical differentials transform by means of a matrix depending only on the mechanical coordinates. For our present purposes we may take this to be of the simpler form

$$
\left.\begin{array}{rl}
d \bar{x}^{b} & =c_{a}^{b}\left(x^{s}\right) \cdot d x^{a} \\
\bar{x}^{s} & =x^{s}
\end{array}\right\}
$$

In a holonomic reference system all geometric objects are represented as functions of the coordinates $x^{\alpha}$; in a new holonomic system they become functions of the new coordinates instead of the old. In a non-holonomic reference frame, however, we do not have a complete set of new coordinates; we have only a set of new differentials. Thus, relative to a non-holonomic reference system, geometric objects are expressed as functions not only of the new differentials but also of the old coordinates. In general, one must carry the original holonomic coordinates along with the new differentials.

In the present situation, owing to the cylinder condition and the special nature of the non-holonomic transformation (11), as also of (10), the geometric objects may be expressed without the aid of the old coordinate system; for that part of the old coordinate system, namely $x^{s}$, which alone enters the expressions for geometric objects is precisely the part that is transformed holonomically.

Consequently, there will here be nothing in the appearance of the transformed geometric objects to characterize them as being in a non-holonomic reference frame. This allows us to regard all geometric objects in the new, non-holonomic reference frame as belonging to a holonomic system, say with coordinates $\bar{x}^{\lambda}$, in a new space differing from the original Riemannian space of $x^{\alpha}$. There will be a cylinder condition on the $m$ electrical coordinates $\bar{x}^{\epsilon}$ in the new space, and under (11) the $n$ mechanical coordinates $\bar{x}^{\sigma}$ in the new space will be the same as those in the old. That is, $\bar{x}^{\sigma}=x^{s}$, or

$$
\bar{x}^{\sigma}=\delta_{s}{ }^{\sigma} x^{s} .
$$


The metric of the new space will be $\bar{L}_{\lambda \mu}\left(\bar{x}^{s}\right)$, obtained by transforming the original $L_{\alpha \beta}$ according to the usual tensor law. Specifically, if we write (11) as.

$$
d \bar{x}^{\lambda}=c_{\alpha}{ }^{\lambda}\left(x^{s}\right) \cdot d x^{\alpha},
$$

where $c_{\alpha}{ }^{\lambda}$ has the form

$$
c_{\alpha}{ }^{\lambda}=\begin{array}{|c|c|}
\hline c_{a}{ }^{\epsilon}\left(x^{s}\right) & 0 \\
\hline 0 & \delta_{s}^{\sigma} \\
\hline \text { electrical } & \begin{array}{c}
\text { me- } \\
\text { chanical }
\end{array}
\end{array} \text { mechanical }
$$

and if we denote the inverse of $c_{\alpha}{ }^{\lambda}$ by $C_{\lambda}{ }^{\alpha}$, the new metric is given by

$$
\bar{L}_{\lambda \mu}\left(x^{s}\right)=C_{\lambda}{ }^{\alpha} C_{\mu}{ }^{\beta} L_{\alpha \beta}
$$

However, as Kron pointed out, the new'space is no longer Riemannian. For the law of transformation of a connection $^{5}$ is

$$
\bar{\Gamma}_{\mu \nu, \lambda}=C_{\lambda}{ }^{\alpha} C_{\mu}{ }^{\beta} C_{\nu}{ }^{\gamma} \Gamma_{\beta \gamma, \alpha}+L_{\alpha \beta} C_{\nu}{ }^{\gamma} C_{\lambda}{ }^{\alpha}\left(\partial C_{\mu}{ }^{\beta} / \partial x^{\gamma}\right),
$$

whether the transformation is holonomic or not. In the present case $\Gamma_{\beta \gamma, \alpha}$ are Christoffel symbols formed from the metric $L_{\alpha \beta}$, namely $[\beta \gamma, \alpha]$. Owing to the special character of the transformation (11) and the cylinder condition on the electrical variables, it is here possible to form Christoffel symbols $\langle[\mu \nu, \lambda]\rangle$ from the new metric $\bar{L}_{\lambda \mu}$. From (15) and (16), and the definition of the Christoffel symbols, it turns out that the original Christoffel symbols $[\beta \gamma, \alpha]$ do not transform into the Christoffel symbols $\langle[\mu \nu, \lambda]\rangle$ but into a connection $\bar{\Gamma}_{\mu \nu, \lambda}$ given by

where

$$
\bar{\Gamma}_{\mu \nu, \lambda}=\langle[\mu \nu, \lambda]\rangle+\Omega_{\mu \lambda \nu}+\Omega_{\nu \lambda \mu}+\Omega_{\mu \nu \lambda}
$$

$$
\Omega_{\lambda \mu \nu}=\frac{1}{2} L_{\alpha \gamma} C_{\nu}{ }^{\gamma}\left(C_{\mu}{ }^{\beta} \frac{\partial C_{\lambda}{ }^{\alpha}}{\partial x^{\beta}}-C_{\lambda}^{\beta} \frac{\partial C_{\mu}^{\alpha}}{\partial x^{\beta}}\right) .
$$

This quantity $\Omega_{\lambda \mu \nu}$ usually depends on the original coordinates $x^{\beta}$. But here, as has already been explained for any geometric object, we may express $\Omega_{\lambda \mu \nu}$ in terms of the new variables $\bar{x}^{\lambda}$. Let us replace $C_{\mu}{ }^{\beta}\left(\partial / \partial x^{\beta}\right)$ by its equivalent $\partial / \partial \bar{x}^{\mu}$, this being valid since we are operating on quantities $C$ which do not.contain electrical variables. Then we may write

$$
\Omega_{\lambda \mu \nu}\left(\bar{x}^{\sigma}\right)=\frac{1}{2} L_{\alpha \gamma} C_{\nu}{ }^{\gamma}\left(\frac{\partial C_{\lambda}^{\alpha}}{\partial \bar{x}^{\mu}}-\frac{\partial C_{\mu}^{\alpha}}{\partial \bar{x}^{\lambda}}\right) .
$$

Under any holonomic transformation on the variables $\bar{x}^{\lambda}$, the quantity $\Omega_{\lambda \mu \nu}$ behaves like an ordinary tensor

${ }^{5}$ We consider the wholly covariant form $\Gamma_{\beta \gamma, \alpha} \equiv L_{\alpha \epsilon} \Gamma_{\beta \gamma}{ }^{\epsilon}$ since its law of transformation contains $C$ but not $c$. There are important occasions in Kron's general theory when singular transformations are employed in which $C$ is defined but not $c$.
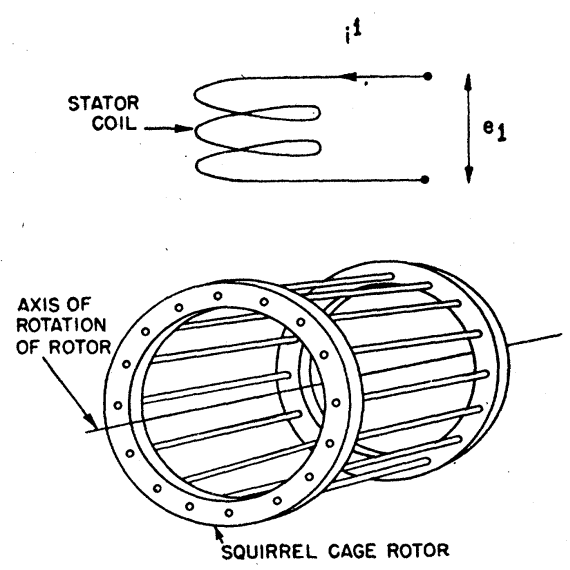

FIG. 1. Single-phase induction motor.

of the third rank, since such a transformation will not affect the index $\alpha$, and for each $\alpha$ the expression in parentheses has the form of a curl.

Thus when we choose to regard the new system as holonomic, we find ourselves in a metric space having a connection given by (17), where the quantity $\Omega_{\lambda \mu \nu}$ behaves like a tensor. This new connection $\bar{\Gamma}_{\mu \nu, \lambda}$ is clearly non-Riemannian. The covariant derivative of the metric with respect to it is still identically zero. For $\bar{\Gamma}_{\mu \nu, \lambda}$ is the transform of the Christoffel symbols $[\beta \gamma, \alpha]$ which satisfy

$$
L_{\alpha \beta, \gamma} \equiv 0,
$$

and under (11) this must transform into

$$
\bar{L}_{\lambda \mu / \nu} \equiv 0 \text {, }
$$

where the solidus denotes the covariant derivative with respect to the new connection $\bar{\Gamma}_{\mu \nu, \lambda}$.

To sum up, we start with a holonomic reference frame in a Riemannian space. When we go over to a non-holonomic reference frame of the special type here discussed, we have the choice of regarding the new system either as a Riemannian space referred to a nonholonomic reference frame or else as a non-Riemannian metrical space, with torsion, referred to a holonomic reference frame.

\section{APPLICATION TO SQUIRREL CAGE INDUCTION MOTOR}

To show how non-holonomic transformations of the type discussed above enter in practice, we discuss the simple case of a single phase squirrel cage induction motor. The generalization to more complicated situations is straightforward; but the basic physical discussion is a little different for phase wound rotors, while the extension to commutator machines introduces special problems.

A single phase squirrel cage induction motor is illustrated symbolically in Fig. 1. It consists of two parts : a stator coil and a cylindrical rotor, the shape of the latter being best described by its name-squirrel cage. The rotor is free to rotate about its cylindrical axis. The stator remains stationary, and into it is fed an alternating electromotive force. For clarity the stator coil has been drawn in the wrong position. In practice it is larger than the 


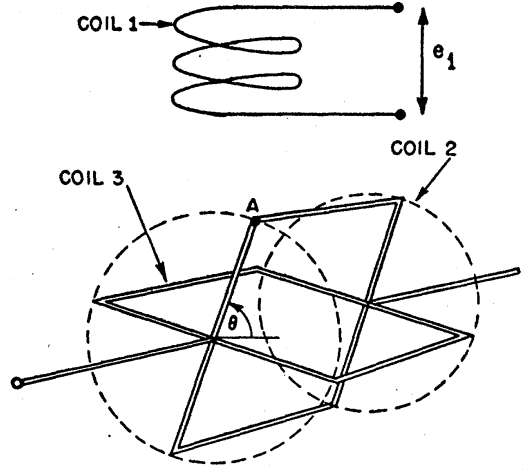

FIG. 2. Moving current paths on moving rotor.

rotor and circumscribes the latter. The cross-bars of the squirrel cage are welded to the end rings and there is no metallic electrical connection between rotor and stator, the stator influencing the rotor only by electromagnetic induction. The rotor is rigidly connected to its own axis of rotation, but this connection is omitted from the figure.

The problem is to set up electrodynamical equations of motion for the performance of the machine. In doing this certain simplifying assumptions are made. For example, rather than treat each cross-bar of the rotor separately, engineers assume that the whole squirrel cage may be adequately represented by a pair of mutually perpendicular closed coils, as in Fig. 2. Since the rotor is symmetric there are no preferred positions for these coils, so we may imagine their positions on the rotor determined arbitrarily, say by marks made on the rotor.

The electrodynamical system illustrated in Fig. 2 has four degrees of freedom, three electrical and one dynamical. The corresponding four-dimensional space has coordinates $x^{\alpha}$, where $\alpha$ has the range 1 to 4 . We label the stator coil coil 1 , and the two rotor coils coil 2 and coil 3 respectively, and denote by $\theta$ the angle that one rotor coil makes with the fixed plane through the axis of rotation perpendicular to the axis of the stator. Then we may write

$$
x^{\alpha}=\left(x^{1}, x^{2}, x^{3}, x^{4}\right)
$$

where $x^{1}, x^{2}, x^{3}$ are the total amounts of charge transported respectively in the circuits 1,2 , and 3 from time $t_{0}$ to any time $t$, and $x^{4}$ stands for the geometrical coordinate $\theta$. The corresponding velocities are

$$
\dot{x}^{\alpha}=\left(i^{1}, i^{2}, i^{3}, \dot{\theta}\right),
$$

where the $i$ 's are the currents in the three circuits and $\dot{\theta}$ is the angular velocity of the rotor.

The dissipation tensor is diagonal and has the form

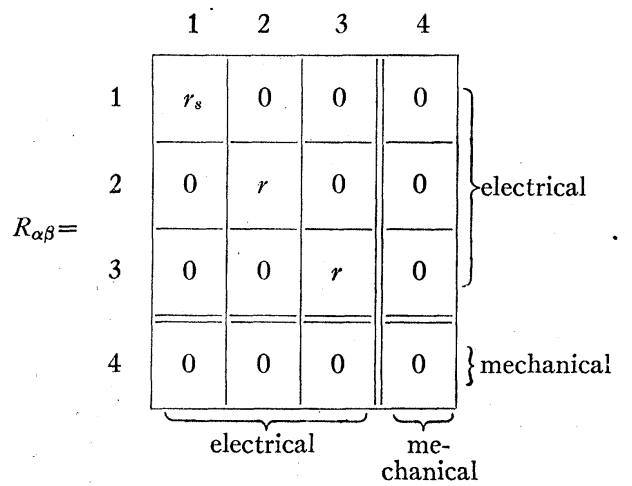

Here $r_{s}$ is the resistance of the stator winding, and $r$ is the re- sistance of each of the rotor coils 2 and 3, these having equal resistances on grounds of symmetry.

The metrical tensor has the general form

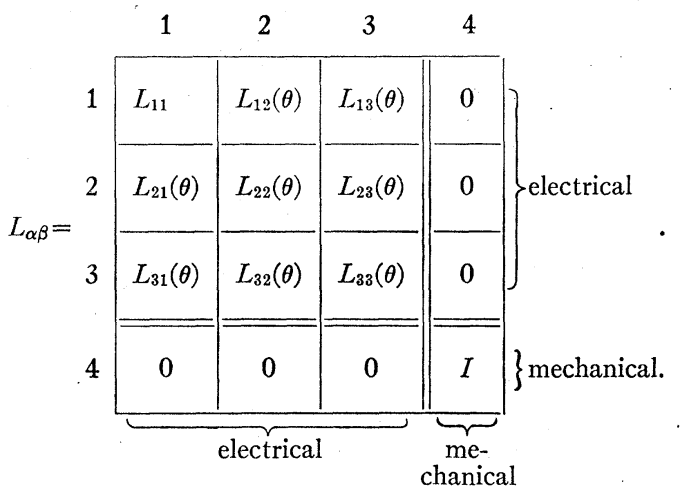

Here the constant $I$ is the moment of inertia of the rotor about its axis, $L_{11}$ is the self inductance of the stator winding, which is a constant, and $L_{12}, L_{13}$ are the mutual inductances between the stator and the respective rotor coils. $L_{22}, L_{33}$ are the self inductances of the rotor coils, and $L_{23}$ their mutual inductance. Let us pretend that the stator has a salient pole. Even though induction motors are not so constructed other motors do have such poles. The salient pole introduces an asymmetry in the air gap between rotor and stator. Were the air gap symmetric $L_{23}$ would be zero, and $L_{22}$ would equal $L_{33}$. But the presence of iron influences inductances, and so for the present we shall leave these $L$ 's as unspecified functions of $\theta$.

If the e.m.f. impressed on the stator winding is denoted by $e_{1}$, and the mechanical torque load imposed on the rotor is denoted by $G$, the generalized force vector is

$$
F_{\alpha}=\left(e_{1}, 0,0, G\right) \text {. }
$$

The zeros enter because no e.m.f.'s are impressed on the rotor coils, induced e.m.f.'s being taken care of by the inclusion of the inductances.

It is now a simple matter to substitute the above values in the Lagrange-Maxwell equations

$$
\frac{d}{d t}\left(\frac{\partial T}{\partial \dot{x}^{\alpha}}\right)-\frac{\partial T}{\partial x^{\alpha}}+\frac{\partial R}{\partial \dot{x}^{\alpha}}=F_{\alpha}
$$

where

and

$$
\begin{gathered}
T=\frac{1}{2} L_{\alpha \beta} \dot{x}^{\alpha} \dot{x}^{\beta} \\
R=\frac{1}{2} R_{\alpha \beta} \dot{x}^{\alpha} \dot{x}^{\beta} .
\end{gathered}
$$

This will give us the equations of motion for the machine. They will be of two types, electrical and mechanical. The former, called the equations of voltage, arise when $\alpha$ has the values 1, 2, 3. The latter, called the equation of torque, is that for which $\alpha$ has the value 4 . If proper assumptions were made concerning the functional forms of the various inductances the equations of motion would be ready for solution. The procedure just outlined pertains to a holonomic reference system in a Riemannian space.

Now, however, let us approach the matter from a different point of view. In replacing the squirrel cage rotor by a pair of mutually perpendicular coils which rotate with the rotor as if rigidly attached to it we were essentially picking out two mutually perpendicular current paths on the rotor and endowing them with such inductances and resistances as would enable them to represent, with sufficient accuracy, the electrical properties of the rotor as a whole. Suppose now that we represent the electrical properties of the rotor by two mutually perpendicular current paths much as before, but with the important difference that they shall be stationary. Such current paths, or coils as we shall call them, will not be rigidly attached to the rotor and we cannot imagine them to be made out of some particular portion of the 
metal forming it. On the contrary, we must imagine a continual metallic metabolism to be taking place in the constitution of these coils. The coils are fixed, but their metallic constitution changes as the rotor rotates.

While this change of viewpoint may seem artificial in the case of a squirrel cage induction motor, it is often essential in more complicated situations. For instance, when a motor has stationary brushes these define stationary metabolistic current paths on the rotor that are of fundamental importance. Even in the present simple case we shall be able to see some of the significance of the new viewpoint.

If we neglect the minor fluctuations arising from the existence of gaps between successive bars of the squirrel cage, we must expect all the inductances of our stationary rotor coils to be constants. Engineers naturally prefer constants to more general functions. They call them design constants, and determine their numerical values either by actual measurement on the machine under investigation or by calculation from a detailed drawing of it.

The present machine, with its stationary rotor coils, has four degrees of freedom. Denote the corresponding coordinates by $\bar{x}^{\lambda}$. The coordinates $\bar{x}^{1}, \bar{x}^{4}$ have the same meaning as $x^{1}, x^{4}$ before. Thus if we take the new, stationary coils $\overline{2}, \overline{3}$ in the position shown in Fig. $3, \bar{x}^{4}$ measures the angle, $\theta$, between coil $\overline{2}$ and the previous coil 2 , and likewise between the coil $\overline{3}$ and the previous coil 3 . The coordinates $\bar{x}^{2}, \bar{x}^{3}$ refer to the stationary coils $\overline{2}, \overline{3}$ and are such that the corresponding "velocities" $\dot{\bar{x}}^{2}, \dot{\bar{x}}^{3}$ are the currents $i^{2}, i^{3}$ flowing in those coils.

The metrical tensor must here be

\begin{tabular}{cc|c|c|c|c|}
$\overline{1}$ & $\overline{1}$ & $\overline{2}$ & $\overline{3}$ & $\overline{4}$ \\
\hline $\bar{L}$ & $\overline{L_{11}}$ & $\bar{L}_{12}$ & 0 & 0 \\
\hline $\bar{L}_{\lambda \mu}=$ & $\bar{L}_{21}$ & $\bar{L}_{22}$ & 0 & 0 \\
\hline$\overline{3}$ & 0 & 0 & $\bar{L}_{33}$ & 0 \\
\hline$\overline{4}$ & $\overline{0}$ & \\
\hline 0 & 0 & 0 & $I$ \\
\hline
\end{tabular}

where all the $\bar{L}$ 's are constants. There will be no mutual inductance between the $\overline{2}$ and $\overline{3}$ coils even if a salient pole does thread the $\overline{1}$ coil on the stator, for we are taking $\overline{3}$ in line with the salient pole and $\overline{2}$ perpendicular to it. However, the presence of the salient pole makes $\bar{L}_{22}$ and $\bar{L}_{33}$ unequal even though the rotor is symmetric. It was in order to show the effect of this type of inequality that we pretended there was a salient pole.

To find the relationship between the new reference system $\bar{x}^{\lambda}$ and the former reference system $x^{\alpha}$ we make a simplifying assumption. The mutual inductance between two plane circuits has its greatest numerical value when they are parallel and falls to zero when they are perpendicular. Although the actual mutual inductance is a complicated function of the angle between the two circuits, it is a standard engineering approximation that near the centers of the coils the mutual inductance varies as the cosine of this angle. We make that assumption here. Since the mutual inductance of two circuits measures the magnetic flux threading one circuit when unit current flows in the other, this assumption amounts to taking the relevant parts of the magnetic fields of the plane coils to be uniform and perpendicular to the planes of the coil.

We wish the pair of coils $\overline{2}, \overline{3}$ to have the same electrical effect as the pair of coils 2, 3. Therefore, for instance, we want the magnetic flux through circuit $\overline{2}$ due to the currents in $\overline{2}$ and $\overline{3}$ to be the same as the magnetic flux through this same circuit $\overline{2}$ due to the currents 2 and 3 . The former flux is measured by $\vec{L}_{22} i^{2}$. The latter is measured by $\left(L_{2 \overline{2}} \bar{i}^{2}+L_{3 \overline{2}} i^{3}\right)$, where $L_{2 \overline{2}}, L_{32}$ are the mutual inductances with $\overline{2}$ of 2 and 3 , respectively.

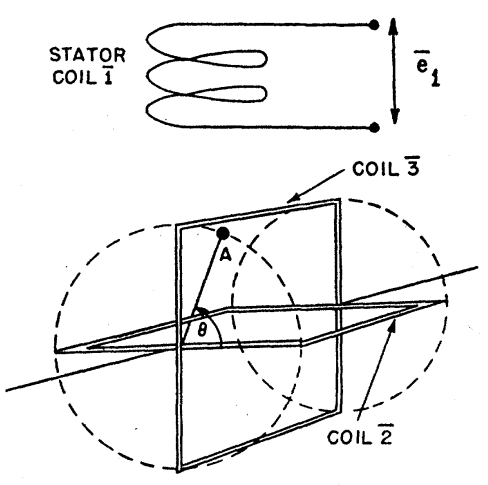

FIG. 3. Stationary current paths on moving rotor.

By the simplifying assumption above, since all the circuits in question have the same shape, we have

$$
\left.\begin{array}{l}
L_{2 \overline{2}}=\bar{L}_{\overline{2} \overline{2}} \cos \theta \\
L_{3 \overline{2}}=\bar{L}_{2 \overline{2}}^{-} \cos \left(\theta+90^{\circ}\right)=-\bar{L}_{\overline{2} \overline{2}} \sin \theta .
\end{array}\right\}
$$

Hence

$$
\bar{L}_{\overline{2} \overline{2}} \overline{i^{2}}=\bar{L}_{\overline{2} \overline{2}} \bar{i}^{2} \cos \theta-\bar{L}_{\overline{2} \bar{i}} i^{3} \sin \theta
$$

or, since $\bar{L}_{\overline{2} 2}$ is not zero,

$$
\overline{i^{2}}=i^{2} \cos \theta-i^{3} \sin \theta .
$$

Similarly, by considering the flux through $\overline{3}$, we find that

$$
\overline{i^{3}}=i^{2} \sin \theta+i^{3} \cos \theta \text {. }
$$

Combining (32) and (33), and the relations $\bar{i}^{\overline{1}}=i^{1}, \bar{\theta}=\theta$, we have

where

$$
\dot{\bar{x}}^{\lambda}=c_{\alpha}{ }^{\lambda} \dot{x}^{\alpha},
$$

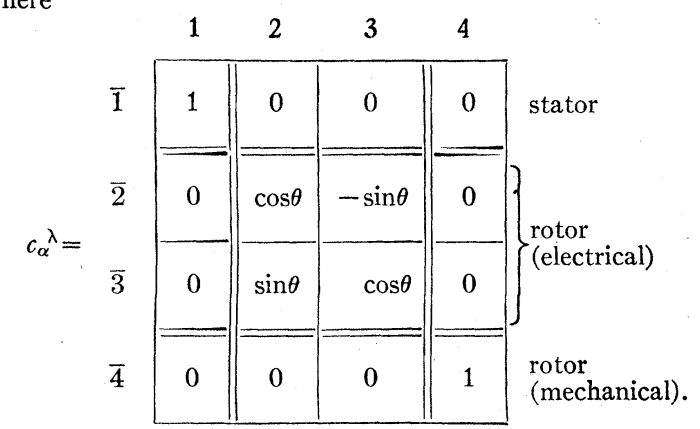

This is a tensor transformation on the velocity vector $\dot{x}^{\alpha}$. And since we require the kinetic energy to be the same whether we use coils 2,3 , or coils $\overline{2}, \overline{3}$, we see from (27) that $L_{\alpha \beta}$ must here transform as a covariant tensor of the second rank; that is

$$
\bar{L}_{\lambda \mu}=C_{\lambda}{ }^{\alpha} C_{\mu}{ }^{\beta} L_{\alpha \beta}
$$

$C_{\lambda}{ }^{\alpha}$ being inverse to $c_{\alpha}{ }^{\lambda}$. Since $c_{\alpha}{ }^{\lambda}$ is orthogonal, $C_{\lambda}{ }^{\alpha}$ has the same matrix as $c_{\alpha}{ }^{\lambda}$.

Now $L_{\alpha \beta}$ and $\dot{x}^{\alpha}$ are tensors in virtue of the role they play in the Lagrange-Maxwell equations, so that if the $\dot{x}$ 's undergo a tensor transformation of the type (34), (35) the $L$ 's must undergo the corresponding tensor transformation (36). We could always make the tensor transformation (34), (35), (36); but without some such discussion as that above we should have no way of interpreting it as a transition to stationary coils $\overline{2}, \overline{3}$, and thus no grounds for taking all the new components $\vec{L}_{\lambda \mu}$ to be constants. Were it not for the simplifying assumptions made, particularly that the mutual inductances varied as the cosine of the angle between the coils, the transformation (34), (35), (36) would not correspond to a transition to stationary coils $\overline{2}, \overline{3}$. Nor would a transition to stationary coils $\overline{2}, \overline{3}$ correspond to a tensor trans- 
formation, in general. However, in engineering it is usually sufficiently accurate to make the above simplifying assumptions, and when greater accuracy is desired the engineer introduces harmonics. Kron's tensor theory is valid for each harmonic.

Let us return to the transformation (34), (35) on the $\dot{x}$ 's. This is essentially a relation between differentials :

$$
\text { Since, by (35), }
$$

$$
d \bar{x}^{\lambda}=c_{\alpha}{ }^{\lambda} d x^{\alpha} .
$$

$$
\partial c_{\alpha}{ }^{\lambda} / \partial x^{\beta} \neq \partial c_{\beta}{ }^{\lambda} / \partial x^{\alpha},
$$

the transformation is non-holonomic. Consequently we must use Kron's non-Riemannian form of the Lorentz-Maxwell equations. These equations may be written

$$
\bar{L}_{\lambda \nu} \dot{\bar{x}}^{\nu}{ }_{\mu \mu} \dot{\bar{x}}^{\mu}+\bar{R}_{\lambda \mu} \dot{\bar{x}}^{\mu}=\bar{F}_{\lambda}
$$

the solidus denoting the covariant derivative with respect to the non-Riemannian connection $\bar{\Gamma}_{\lambda \mu, \nu}$ defined in (17) and (18) or (19) and (22). Owing to the antisymmetry of $\Omega_{\lambda \mu \nu}$ in the first two indices, $\left(4^{\prime}\right)$ may be written

$$
\bar{L}_{\lambda \nu} \ddot{\bar{x}}^{\nu}+[\mu \nu, \lambda] \dot{\bar{x}}^{\mu} \dot{\bar{x}}^{\nu}+2 \Omega_{\mu \lambda \nu} \dot{\bar{x}}^{\mu} \dot{\bar{x}}^{\nu}+\bar{R}_{\lambda \mu} \dot{\bar{x}}^{\mu}=\bar{F}_{\lambda},
$$

which is the same as

$$
\frac{d}{d t}\left(\frac{\partial \bar{T}}{\partial \dot{\bar{x}}^{\lambda}}\right)-\frac{\partial \bar{T}}{\partial \bar{x}^{\lambda}}+2 \Omega_{\mu \lambda \nu} \dot{x}^{-\mu} \dot{x}^{-\nu}+\frac{\partial \bar{R}}{\partial \dot{\bar{x}}^{\lambda}}=\bar{F}_{\lambda} .
$$

Comparing $\left(1^{\prime}\right)$ with (1) or (26) we see that the essential difference is the entry of the additional term $2 \Omega_{\mu \lambda \nu} \dot{\bar{x}}^{\mu} \dot{\bar{x}}^{\nu}$.

We have

$$
\bar{T}=\frac{1}{2} \bar{L}_{\lambda \mu} \dot{\bar{x}}^{\lambda} \dot{\bar{x}}^{\mu},
$$

where $\bar{L}_{\lambda \mu}$ is given in (29). Also

$$
\bar{R}=\frac{1}{2} \bar{R}_{\lambda \mu} \dot{\bar{x}}^{\lambda} \dot{\bar{x}}^{\mu} .
$$

And from (23) and (35) we see that $\bar{R}_{\lambda \mu}$ has the same components as $R_{\alpha \beta}$. If we used these values of $\bar{T}$ and $\bar{R}$ in the original form of the Lagrange-Maxwell equations, (26), we would obtain wrong results. We must use them in the generalized form $\left(1^{\prime}\right)$. However, the ordinary Lagrange-Maxwell equations, (26), would give correct results if we used the appropriate form (24) of the metrical tensor $L_{\alpha \beta}$. It is of interest to see what the simplifying assumptions made above imply in terms of the form of (24). Transforming from the constant $\tilde{L}_{\lambda \mu}$ back to the $L_{\alpha \beta}$ in the holonomic frame by means of the tensor law

\begin{tabular}{|c|c|c|c|c|}
\hline 1 & $\bar{L}_{\mathrm{ii}}$ & $\bar{L}_{\overline{12}} \cos \theta$ & $-\bar{L}_{\overline{12}} \sin \theta$ & 0 \\
\hline 2 & $\bar{L}_{12} \cos \theta$ & $L_{S}-L_{D} \cos 2 \theta$ & $L_{D} \sin 2 \theta$ & 0 \\
\hline 3 & $-\bar{L}_{12} \sin \theta$ & $L_{D} \sin 2 \theta$ & $L_{S}+L_{D} \cos 2 \theta$ & 0 \\
\hline 4 & 0 & 0 & 0 & $I$ \\
\hline
\end{tabular}

$$
L_{\alpha \beta}=c_{\alpha}{ }^{\lambda} c_{\beta}{ }^{\mu} \bar{L}_{\lambda \mu}
$$

with $c_{\alpha}{ }^{\lambda}$ given by (35), we find that

1

2

3

4

where

$$
L_{S}=\frac{1}{2}\left(\bar{L}_{\overline{2} \overline{2}}+\bar{L}_{\overline{3} \overline{3}}\right), \quad L_{D}=\frac{1}{2}\left(\bar{L}_{\overline{2} \overline{2}}-\bar{L}_{\overline{3} \overline{3}}\right) .
$$

If the air gap is symmetric, $\bar{L}_{\overline{2} \overline{2}}=\bar{L}_{\overline{3}} \overline{3}$, and so $L_{D}$ is zero. In this case the components $L_{23}, L_{32}$ of $L_{\alpha \beta}$ vanish and this means there is no mutual inductance between the moving coils 2,3 on the rotor. When there is a salient pole or other asymmetry in the air gap this mutual inductance will not vanish.

The above has touched on only one of the many aspects of Kron's work. His various tensor ideas have a wide range of application. They cover such things as the theory of stationary electrical networks, the interconnection of complex systems, whether electrodynamical, mechanical, or hydrodynamical, the hunting ${ }^{6}$ and stability of all such systems, and the setting up of equivalent circuits not only for their performance under different conditions of operation but even for the partial differential equations of classical and quantum physics.

\footnotetext{
${ }^{6}$ The engineering term for small oscillations about steady
} motion. 